\title{
Kinderradiologen starten digitale Forschungsplattform zur besseren \\ Experten-Vernetzung
}

Kinderonkologische Fragestellungen machen den Anfang - Tübinger Kinderradiologe Prof. Schäfer steht neuer Forschungskommission der Gesellschaft für Pädiatrische Radiologie e. V. vor

Sogenannte Seltene Erkrankungen sind bei Kindern tatsächlich gar nicht so selten. Dennoch reichen die medizinischen Daten, die im Rahmen der Diagnostik und Therapie dieser jungen Menschen erhoben werden, selbst in einem größeren Behandlungszentrum - einer Universitätsklinik oder einem Kinderkrankenhaus - in der Regel nicht aus, um hierauf sinnvolle Forschungsprojekte aufzubauen.

Multicenterstudien sind daher erforderlich. Aus diesem Grund hat die Gesellschaft für Pädiatrische Radiologie (GPR e.V.), die deutschsprachige Fachgesellschaft der Kinder- und Jugendradiologen, eine Forschungskommission mit dem Ziel einer digitalen Plattform gegründet, die das Sammeln radiologischer Bilddaten, ihre Auswertung und den kollegialen Austausch über das zusammengengetragene Datenmaterial ermöglichen soll.

Die Federführung des Projekts liegt bei dem forschungserfahrenen Kinder- und Jugendradiologen Prof. Dr. Jürgen Schäfer vom Universitätsklinikum Tübingen. Die
Gesellschaft für Pãdiatrische Radiologie e.V.

technische Basis stellt die Firma mbits imaging $\mathrm{GmbH}$ aus Heidelberg, die seit Jahren Lösungen für den datensicheren Austausch radiologischer Bilder anbietet.

„Mit dieser Forschungsplattform können sich künftig universitäre, aber auch nichtuniversitäre Kinder- und Jugendradiologinnen und -radiologen vernetzen, um Daten, Studienergebnisse und innovative Methoden zu teilen. Sie können so in gemeinsamen Projekten und viel effizienter als bisher seltene Erkrankungen unter radiologischen Gesichtspunkten untersuchen. Ziel dieser Zusammenarbeit ist es, sowohl neue Erkenntnisse für die bildgebende Diagnostik und Therapie zu gewinnen als auch bereits bekannten radiologischen Parametern und Biomarkern mehr Evidenz zu verleihen und diese im klinischen Alltag nutzbar zu machen“, erklärt Professor Schäfer.

Eines der ersten Projekte, das auf der Plattform abgebildet wird, befasst sich mit radiologischen Bilddaten zur Primärdiagnostik und zum Therapieansprechen bei der Behandlung des Neuroblastoms, einem der häufigsten kindlichen Tumoren. Weitere Projekte sind in Arbeit.

\section{Was ist Kinderradiologie?}

Die Kinder- und Jugendradiologie (offizielle Bezeichnung durch die Bundesärztekammer seit 2019) beschäftigt sich mit der speziellen Bildgebung beim Neugeborenen, Säugling, Kleinkind, Schulkind und Jugendlichen.

Kinderradiologinnen und -radiologen sind Radiologinnen und Radiologen, die in einer (in Deutschland aktuell 2-jährigen) fachärztlichen Schwerpunktweiterbildung speziell in der Kinderradiologie geschult sind. Sie legen großen Wert darauf, die Untersuchungen von Kindern schonend und mit geringstmöglicher Strahlendosis durchzuführen. Bevorzugte Methoden kinderradiologischer Bildgebung sind daher die ohne Röntgenstrahlen arbeitenden Verfahren wie die Sonografie (Ultraschalluntersuchung) und die Magnetresonanztomografie (MRT).

Die GPR als wissenschaftliche Vereinigung der deutschsprachigen Kinderradiologen (Deutschland, Österreich und Schweiz) wurde 1963 gegründet. Aktuell zählt sie über 380 Mitglieder. Im Fokus steht die wissenschaftliche Vernetzung, die Nachwuchsförderung, die Vertretung des Fachs im Wissenschaftsbetrieb und in der Berufspolitik.

Weitere Informationen erhalten Sie auf: www.kinder-radiologie.org. 